\title{
Consumo de sustancias y características sociodemográficas de solicitantes de tratamiento ambulatorio en una red de atención especializada en México: análisis comparativo entre 2007 y 2014
}

\author{
David Bruno Díaz Negrete', Alma Delia Gutiérrez López', Carmen Fernández Cáceres', Ricardo Sánchez-Huesca' \\ 'Centros de Integración Juvenil, A.C., México
}

\section{RESUMEN}

Introducción: el Sistema de Información Epidemiológica del Consumo de Drogas de Centros de Integración Juvenil integra datos de la población que solicita tratamiento en las 116 unidades que conforman la red nacional de atención. Objetivo: el presente trabajo compara las características sociodemográficas y de consumo en usuarios de drogas solicitantes de tratamiento ambulatorio entre 2007 y 2014. Método: se recuperó información de cerca de 170 mil casos, principalmente de población atendida entre 2007 y 2014 ( $n=18,136$ y 21,320, respectivamente). El análisis comprendió la descripción de tendencias del consumo 2007-2014 en un nivel nacional; la comparación del perfil sociodemográfico y el patrón de consumo de la población atendida en este periodo; así como la comparación regional del uso reciente de sustancias y de su crecimiento medio anual. Resultados: se observa un aumento significativo del consumo de mariguana, metanfetaminas, alucinógenos y éxtasis, mientras que el uso de cocaína, inhalables y heroína presenta una disminución. Se registra un incremento de mujeres y menores de edad atendidos, una menor edad de inicio y un aumento del consumo de mariguana como droga de inicio y de mayor impacto. Finalmente, se aprecian diversas diferencias regionales. Discusión y conclusiones: los resultados confirman lo reportado en encuestas de México respecto al aumento del uso de mariguana y metanfetaminas, así como la disminución del uso de cocaína, inhalables y heroína, contrario a lo reportado en otras poblaciones. Estos hallazgos proporcionan información relevante para el desarrollo de programas y servicios en México.

Palabras clave: monitoreo epidemiológico; abuso de drogas; centros de tratamiento; solicitantes de tratamiento.

\begin{abstract}
Introduction: the Epidemiological Information System on Drug Abuse of Centros de Integración Juvenil, integrates data from all the treatment seekers in 116 units from the national network. Objective: this work aims to compare sociodemographic and drug abuse patterns in illicit drug users seeking outpatient treatment between 2007 and 2014. Method: data was retrieved from about 170,000 cases, from population attending in 2007 and 2014 $(n=18,136$ and 21,320 , respectively). Analysis included the description of 2007-2014 consumption trends at a national level; comparison of sociodemographic characteristics and consumption patterns of patients initiating treatment in 2007 and 2014; and regional comparison of recent substance use and its average annual growth between these last two years. Results: a significant increase of marijuana, methamphetamines, hallucinogens and ecstasy consumption was detected; meanwhile, cocaine, inhalants and heroin use showed a decrease. A higher percentage of women and children seeking treatment, an earlier drug use onset and a higher level of marijuana reported as onset substance and as drug motivating treatment seeking were also observed. Finally, several regional differences were registered. Discussion and conclusions: these results confirm those reported in population surveys carried out in Mexico regarding the increased use of substances as marijuana and methamphetamine; by contrast, the decline in cocaine, inhalants and heroin use is contrary to the reported in other populations. Despite the limitations of the study, its findings may provide significant information to develop prevention and treatment programs in Mexico.
\end{abstract}

Keywords: epidemiological monitoring; drug abuse; drug treatment centers; treatment seekers.

Autor de correspondencia:

David Bruno Díaz Negrete. Subdirección de Investigación, Centros de Integración Juvenil, A.C. Tlaxcala núm.208, col. Hipódromo, del. Cuauhtémoc, C.P. 06100, Distrito Federal, México. Tel.:+52(55) 5999 7724. Correo electrónico: investigacion@cij.gob.mx.

Recibido: 04 de agosto del 2015.

Aceptado: 18 de septiembre del 2015

DOI: $10.28931 /$ riiad.2015.1.03 


\section{INTRODUCCIÓN}

México cuenta con un amplio sistema de información epidemiológica que comprende encuestas poblacionales, estudios con grupos ocultos y sistemas de información en centros de tratamiento e instituciones de salud y procuración de justicia, lo que contribuye a configurar, de acuerdo con las recomendaciones internacionales, un diagnóstico integrado del problema de las adicciones.

A través de este sistema se han logrado conocer oportunamente la distribución, la magnitud, la forma y las tendencias del consumo de drogas, así como los grupos, factores de riesgo y problemas emergentes, con lo que ha sido posible establecer prioridades para la aplicación de servicios y la definición de políticas públicas, así como generar posibles indicadores para la evaluación del impacto de programas (Programa de las Naciones Unidas para la Fiscalización Internacional de Drogas [PNUFID], 2003).

En particular, los sistemas de información en centros de tratamiento constituyen un mecanismo útil para el seguimiento de las características epidemiológicas del consumo en grupos afectados por el problema (Programa Mundial de Evaluación del Uso Indebido de Drogas [GAP], Observatorio Europeo de las Drogas y las Toxicomanías [EMCDDA, por sus siglas en inglés], 2006; Díaz, Sánchez, \& Guisa, 2004). Desde hace 46 años, Centros de Integración Juvenil, A.C. (CIJ) realiza actividades de prevención, tratamiento, rehabilitación e investigación científica sobre el consumo de drogas en México, además de formar recursos humanos especializados en la atención de esta problemática; cuenta con un Sistema Institucional de Información Epidemiológica del Consumo de Drogas (SIECD) que recupera datos de la totalidad de usuarios que solicitan los servicios de tratamiento en las 116 unidades que conforman la red nacional de atención.

A partir del reconocimiento de la importancia de contar con información válida y actualizada para apoyar el diseño y la aplicación de programas, ClJ ha reportado desde 1979 datos del patrón de consumo de sustancias entre pacientes atendidos (Sánchez \& López, 1985). Dicha información fue recuperada y sistematizada a lo largo de la década de 1980 (Clarac \& Wagner, 1987), lo que permitió establecer, a partir de 1990, indicadores sociodemográficos y patrones de consumo comparables, que se han ajustado conforme a las necesidades de información emergentes, para desembocar en la conformación actual del SIECD (CIJ, 2015).

A pesar de los avances logrados en materia de sistemas de información epidemiológica, los datos sobre el crecimiento de la demanda de servicios de atención del consumo de drogas son escasos, con lo cual se limita el conocimiento de los cambios en la utilización de servicios a través del tiempo.
Con base en el análisis de información del SIECD, el presente trabajo tiene dos objetivos: describir y comparar las características demográficas y del consumo de sustancias en usuarios de drogas que solicitaron tratamiento en unidades ambulatorias de la red de tratamiento de ClJ entre 2007 y 2014; y determinar los cambios en el número de usuarios de drogas que solicitaron atención en dichas unidades, con el propósito de describir el aumento o disminución del uso de sustancias a nivel nacional y regional.

\section{MÉTODO}

\section{Población y muestra}

Se recuperó información de usuarios de drogas ilícitas, solicitantes de tratamiento por primera vez en el periodo 2007-2014, en unidades de consulta externa de la red nacional de atención de ClJ; el total acumulado fue de 169 mil 546 casos (las unidades en las que fueron captados aumentaron de 79 en 2007 a 95 en 2014). El presente estudio se concentra en la población atendida en los años 2007 y 2014, a nivel nacional y regional, con regiones establecidas de acuerdo con las propuestas de la Encuesta Nacional de Adicciones (Villatoro et al., 2012a).

\section{Recolección y recuperación de datos}

La información del SIECD es obtenida en entrevista inicial, realizada de manera individual como parte del procedimiento de ingreso al tratamiento y registrada en formato electrónico directamente por los terapeutas responsables de la recepción de pacientes en las unidades de la red de atención. Con base en esto se conforma una base de datos nacional integrada y validada en cortes semestrales, de la cual se toman datos anualizados.

\section{Mediciones}

Las variables de estudio comprenden datos sociodemográficos como sexo, edad, estado civil, escolaridad y ocupación; uso de sustancias alguna vez en la vida y en el mes previo a la solicitud de tratamiento; edad de inicio y drogas ilícitas de inicio; sustancia de mayor impacto o que ha motivado la búsqueda de tratamiento; uso combinado de sustancias (incluyendo alcohol); frecuencia de la utilización de drogas ilícitas en el mes anterior; y tiempo transcurrido entre el inicio del consumo y la solicitud de tratamiento.

Las sustancias consideradas comprenden alcohol, tabaco, cannabis (mariguana y hashish), inhalables (solventes y removedores, pegamentos, esmaltes y pinturas, entre otros), cocaína (clorhidrato de cocaína) y crack (cocaína 
de base libre), tomadas separadamente en virtud del interés clínico que representan y para apoyar la planeación de tratamientos específicos por región; además se abordan las metanfetaminas (cristal, hielo), DMDA y otras metanfetaminas alucinógenas (reportadas, de manera genérica, bajo el nombre de éxtasis, por tratarse de la sustancia de mayor uso en este grupo), las benzodiacepinas (incluido Rohypnol), los alucinógenos (principalmente LSD, plantas alucinógenas y derivados) y la heroína.

\section{Análisis estadístico}

Se realizó un análisis descriptivo de las tendencias observables entre 2007 y 2014 a nivel nacional, tanto en el consumo de drogas alguna vez en la vida como en el mes previo a tratamiento.

Posteriormente se comparó el perfil sociodemográfico y el patrón de consumo de la población atendida en 2007 y 2014 a nivel nacional. Conforme a los parámetros de estudios con población solicitante de tratamiento (Faller et al., 2014; Ponizovsky, Rosca, Haklai, \& Goldberger, 2015), los datos nominales se presentan en términos de porcentajes, en tanto que las variables numéricas se reportan mediante la mediana y el rango intercuartil. En congruencia con esto, se aplicaron, para la identificación de diferencias significativas (con p>.05), las pruebas de Ji cuadrada y Kolmogorov-Smirnov para dos muestras independientes. Para este efecto, se utilizó el programa SPSS v. 19.

Por último, con el fin de contar con una estimación del aumento o disminución del uso de sustancias en la categoría de uso en el mes previo a la solicitud de tratamiento a nivel nacional y en las regiones establecidas para este propósito, se calculó la tasa media de crecimiento anual real (TMCAR) entre 2007 y 2014, controlando la tasa media de crecimiento anual de los ingresos a tratamiento en el periodo.

\section{RESULTADOS}

\section{Distribución de usuarios de drogas por región}

En la Tabla 1 se muestra la distribución por región de las entidades federativas en las que fue captada la población de este estudio, el total de ingresos registrados entre 2007 y 2014 en los niveles nacional y regional, así como la correspondiente tasa media de crecimiento anual (TMCA). Se observa, en general, un aumento de la población atendida, excepto en la región Nororiental donde se registra una contracción.

Tabla 1

Distribución de usuarios de drogas ilícitas solicitantes de tratamiento en unidades de Centros de Integración Juvenil agrupadas por región, en 2007 y 2014: ingresos y tasa media de crecimiento anual

\begin{tabular}{|c|c|c|c|c|}
\hline Región & Entidades federativas comprendidas & 2007 & 2014 & $\mathrm{TMCA}^{\mathrm{a}}$ \\
\hline Región Noroccidental & Baja California, Baja California Sur, Sinaloa y Sonora. & 2,128 & 2,861 & .04 \\
\hline Región Norcentral & Coahuila, Chihuahua y Durango. & 1,516 & 1,590 & .01 \\
\hline Región Nororiental & Nuevo León, San Luis Potosí y Tamaulipas. & 1,478 & 1,225 & -.03 \\
\hline Región Occidental & Aguascalientes, Colima, Jalisco, Nayarit y Zacatecas. & 2,453 & 3,112 & .03 \\
\hline Región Centro & $\begin{array}{l}\text { Guanajuato, Hidalgo, Estado de México, Morelos, Puebla, } \\
\text { Querétaro y Tlaxcala. }\end{array}$ & 4,024 & 4,724 & .02 \\
\hline $\begin{array}{l}\text { Ciudad de México } \\
\text { (Distrito Federal) }\end{array}$ & & 4,330 & 4,897 & .02 \\
\hline Región Centro Sur & Guerrero, Michoacán, Oaxaca y Veracruz. & 1,060 & 1,392 & .04 \\
\hline Región Sur & Campeche, Chiapas, Quintana Roo, Tabasco y Yucatán. & 1,147 & 1,519 & .04 \\
\hline Nacional & & 18,136 & 21,320 & .02 \\
\hline
\end{tabular}

a Tasa media de crecimiento anual. 


\section{Tendencias del consumo de sustancias a nivel nacional}

En el periodo estudiado, el uso de mariguana a lo largo de la vida aumentó más de 16 puntos porcentuales, situándose por arriba del uso de alcohol y tabaco (Figura 1). Se aprecia, del mismo modo, un incremento del consumo de metanfetaminas de cerca de seis puntos entre 2010 y 2014, tras una disminución registrada en los primeros años del periodo. En un menor nivel de prevalencia, tanto el uso de alucinógenos como el de éxtasis presentan tendencias al alza.

Por el contrario, el consumo de cocaína, crack, inhalables, benzodiacepinas y heroína registra una tendencia decreciente. En el caso de la cocaína y el crack, el declive es de más de 20 puntos porcentuales, mientras que el uso de inhalables presenta, entre 2011 y 2014 , un descenso de cerca de 13 puntos, después de haber mostrado un aumento en los primeros años del periodo estudiado, para situarse en 2014 casi en el mismo nivel que la cocaína. Por último, la disminución del consumo de benzodiacepinas y heroína entre 2007 y 2014 es de tres y un punto, respectivamente.
Las tendencias del consumo de sustancias en los 30 días previos al inicio del tratamiento son similares a las registradas en la categoría de uso alguna vez en la vida. Se aprecia un alto nivel de consumo de alcohol y tabaco, con una ligera tendencia a la baja; un acusado crecimiento del uso de mariguana, hasta igualar al alcohol; un incremento del reporte de inhalables y metanfetaminas, con un posterior decremento en el caso de sustancias inhalables; así como una disminución en la utilización de cocaína y crack. Como excepción, el uso actual de benzodiacepinas registra un aumento, aunque su utilización alguna vez en la vida tiende a disminuir (Figura 2).

\section{Diferencias entre la población atendida en 2007 y 2014}

Las tendencias descritas están acompañadas por diversos cambios en el perfil sociodemográfico de la población, así como en otros indicadores de consumo.

A partir de la comparación de la población recibida en tratamiento en 2007 y 2014, se observa una disminución relativa del número de hombres y un aumento de

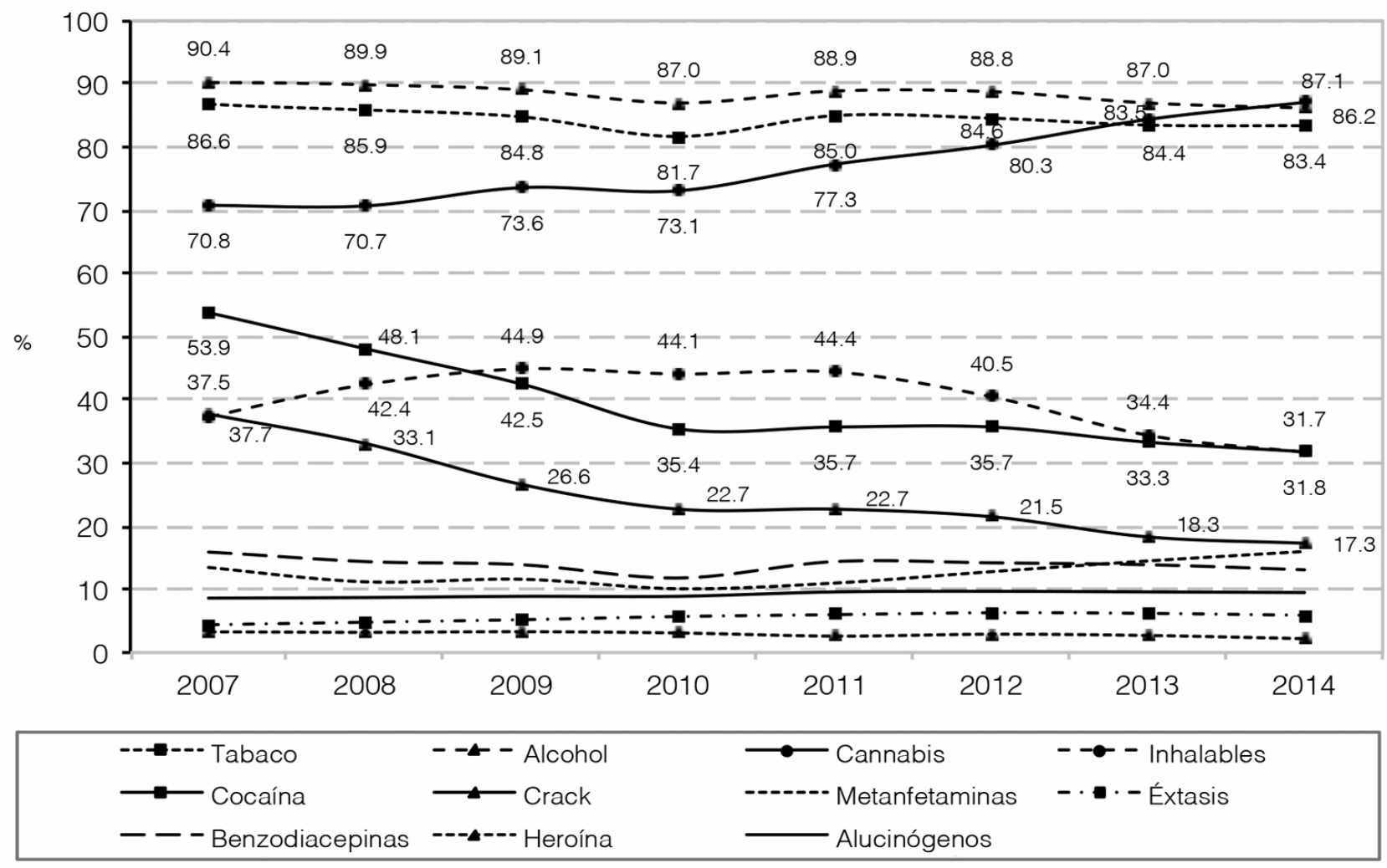

Figura 1. Tendencias del consumo de sustancias alguna vez en la vida, usuarios de drogas ilícitas solicitantes de tratamiento en Centros de Integración Juvenil de 2007 a 2014, nivel nacional 
mujeres, al pasar de una tasa de 4.9 hombres por cada mujer, a una tasa de 4.3. Asimismo, la mediana de edad de la población resulta menor en cinco años y, mientras que menos de una tercera parte de la población atendida en 2007 era menor de edad, en 2014 este grupo conformó prácticamente la mitad de la población atendida (Tabla 2).

Del mismo modo, se observa un aumento de la proporción de casos de solteros y estudiantes, así como una diferencia significativa en el nivel de estudios reportado, con un porcentaje mayor de casos que reportan estudios de bachillerato, lo que refleja, en general, el ingreso de pacientes con una mayor escolaridad.

Por otro lado, la población atendida en 2014 refirió una edad mediana un año menor en el inicio del uso de alcohol y de drogas ilícitas. En particular, es posible observar una diferencia en el porcentaje de niños menores de 13 años, 3.2 puntos porcentuales mayor en 2014. Igualmente, se aprecian diferencias en las drogas de inicio, sin considerar alcohol o tabaco, con un porcentaje notoriamente mayor de uso inicial de mariguana y la disminución del uso de inhalables, cocaína y crack (Tabla 3).

Las tendencias del uso de sustancias alguna vez en la vida y en el último mes ya descritas se traducen, salvo excepciones, en la existencia de cambios significativos en las proporciones de uso registradas en 2007 y 2014 , con el aumento ya señalado del uso de mariguana y metanfetaminas, y la disminución de cocaína, crack e inhalables, entre otros cambios. Estas diferencias se ven reflejadas en un claro aumento de la mariguana como droga de mayor impacto, esto es, como sustancia que motiva la búsqueda de tratamiento (una diferencia de cerca de 32 puntos porcentuales entre la población atendida en 2007 y la atendida en 2014) y en la baja correlativa de cocaína y crack, entre otras sustancias (Tabla 4).

Los datos también reflejan la existencia de diferencias en la frecuencia del uso combinado de sustancias en el último año (incluyendo alcohol y drogas ilícitas), la cual registra una disminución significativa. Asimismo, la población recibida en tratamiento en 2014 reporta una utilización considerablemente mayor de drogas ilegales en el mes previo al tratamiento y una asistencia más temprana al mismo (Tabla 5).

\section{Uso de sustancias en el último mes 2007-20I4, por región}

De acuerdo con las tendencias ya descritas, entre 2007 y 2014, el consumo de mariguana en el mes pre-

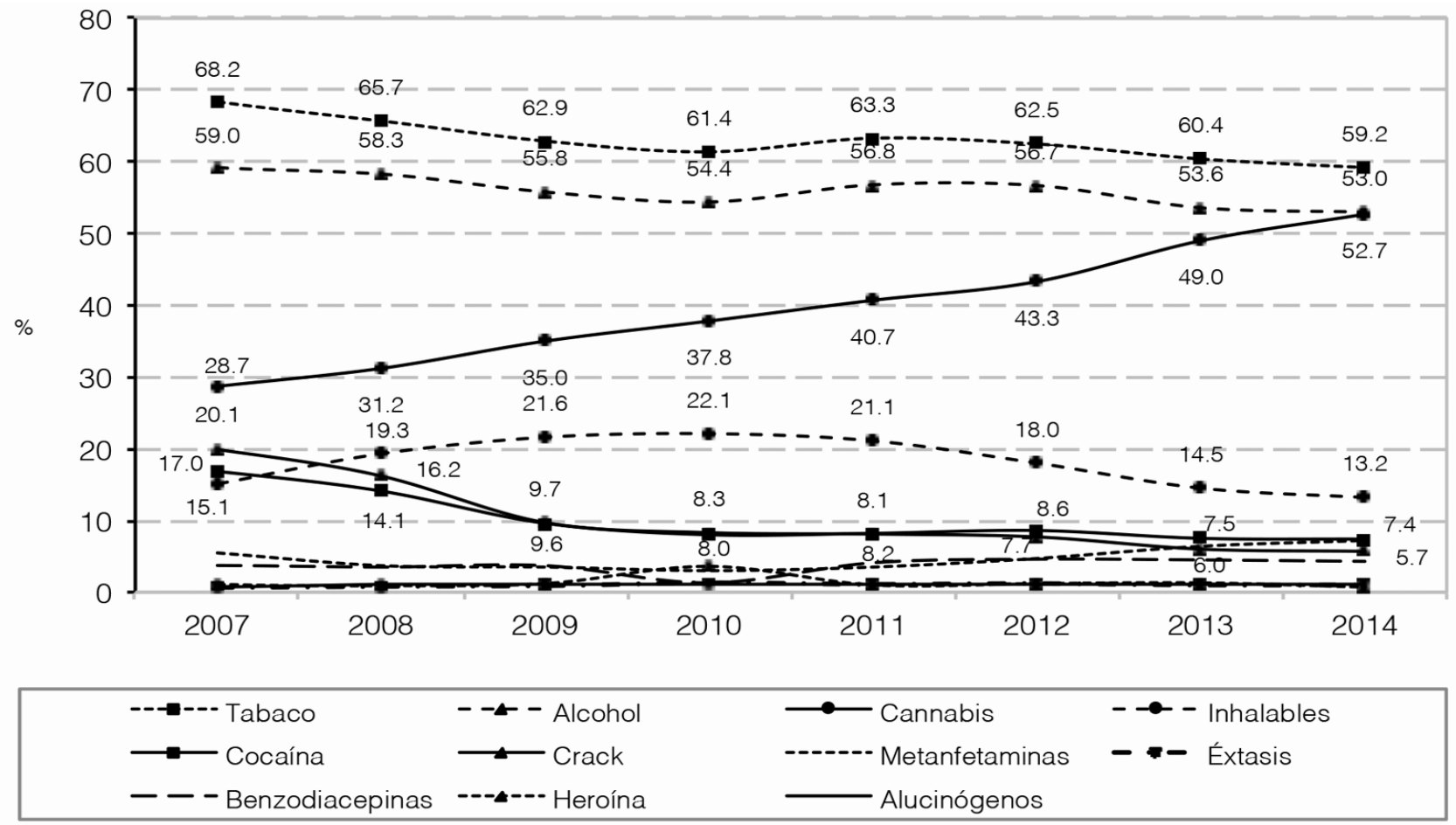

Figura 2. Tendencias del consumo de sustancias en los últimos 30 días, usuarios de drogas ilícitas solicitantes de tratamiento en Centros de Integración Juvenil de 2007 a 2014, nivel nacional 
vio al tratamiento registró, a nivel nacional, una tasa media de crecimiento anual real (TMCAR) de 9.1\%; el de éxtasis, de 6.9\%; el de alucinógenos, de 7.0\%; el de metanfetaminas, de 3.8\%; y el de benzodiacepinas, de $1.8 \%$. En cuanto a las sustancias cuya utilización registra, por el contrario, una disminución, sobresalen la cocaína y el crack, con una contracción promedio de -11.2 y $-16.4 \%$ anual, respectivamente; seguidos por la heroína y los inhalables, con una TMCAR de -2.6 y-1.9\%; así como por el tabaco y el alcohol, con una reducción de -2.0 y $-1.5 \%$ anual. A partir de estos indicadores de aumento-decremento, y de la prevalencia del consumo de sustancias en el último mes a nivel nacional (Tabla 4, columnas centrales), se realiza una caracterización de las diferencias reportadas por la población atendida en las distintas regiones del país (Tabla 6).
En la región Noroccidental se presenta una disminución del uso de casi todas las sustancias, con excepción de la mariguana, cuyo aumento de 5.7\% anual resulta el menor de los observados en todas las regiones del país. La proporción de usuarios de metanfetaminas y de cocaína captados en esta región en 2014 es la más alta del país, mientras que la de consumidores de heroína (captados en su mayoría en las unidades situadas en las ciudades de Tijuana y Mexicali) resulta la segunda más alta.

A la región Norcentral le corresponden los más altos porcentajes de uso reciente de inhalables, benzodiacepinas y heroína registrados en 2014, a pesar de que la heroína, junto con la cocaína y el crack, presenta una tendencia a la disminución. Otras drogas, como los alucinógenos, el éxtasis y las benzodiacepinas presentan altas tasas de aumento en la región, al igual que los inhalables,

Tabla 2

Características sociodemográficas de usuarios de dogas ilícitas solicitantes de tratamiento en Centros de Integración Juvenil en 2007 y 2014

\begin{tabular}{|c|c|c|c|c|}
\hline & & $\begin{array}{c}2007 \\
(\mathrm{~N}=18,136)\end{array}$ & $\begin{array}{c}2014 \\
(N=21,320)\end{array}$ & Sig. \\
\hline \multirow[t]{2}{*}{ Sexo } & Hombres & $83.1 \%$ & $81.2 \%$ & $>.001$ \\
\hline & Mujeres & $16.9 \%$ & $18.8 \%$ & \\
\hline \multirow[t]{3}{*}{ Edad } & Mediana (rango intercuartil) & 23 años (7) & 18 años (5) & $>.001$ \\
\hline & Menores de 18 años & $28.5 \%$ & $49.9 \%$ & $>.001$ \\
\hline & 18 años o más & $71.5 \%$ & $50.1 \%$ & \\
\hline \multirow[t]{6}{*}{ Estado civil } & Soltero(a) & $58.1 \%$ & $75.6 \%$ & $>.001$ \\
\hline & Casado(a) & $19.1 \%$ & $8.8 \%$ & \\
\hline & Unión libre & $13.0 \%$ & $9.2 \%$ & \\
\hline & Separado(a) & $7.8 \%$ & $5.0 \%$ & \\
\hline & Divorciado(a) & $1.7 \%$ & $1.1 \%$ & \\
\hline & Viudo(a) & $.3 \%$ & $.2 \%$ & \\
\hline \multirow[t]{8}{*}{ Escolaridad } & Sin estudios, no sabe leer ni escribir & $.6 \%$ & $.3 \%$ & $>.001$ \\
\hline & Sin estudios, sabe leer y escribir & $.4 \%$ & $.2 \%$ & \\
\hline & Primaria & $15.1 \%$ & $8.1 \%$ & \\
\hline & Secundaria & $45.6 \%$ & $45.2 \%$ & \\
\hline & Estudios técnicos o comerciales & $3.2 \%$ & $2.4 \%$ & \\
\hline & Bachillerato o bachillerato técnico & $26.2 \%$ & $35.9 \%$ & \\
\hline & Estudios superiores & $8.6 \%$ & $7.7 \%$ & \\
\hline & Estudios de postgrado & $.3 \%$ & $.2 \%$ & \\
\hline \multirow[t]{6}{*}{ Ocupación } & Estudiante & $19.9 \%$ & $41.9 \%$ & $>.001$ \\
\hline & Estudiante con actividad laboral & $3.6 \%$ & $4.9 \%$ & \\
\hline & Con actividad laboral & $47.5 \%$ & $28.1 \%$ & \\
\hline & Desempleado(a) & $25.0 \%$ & $21.4 \%$ & \\
\hline & Tareas del hogar & $3.7 \%$ & $3.2 \%$ & \\
\hline & Pensionado & $.2 \%$ & $.5 \%$ & \\
\hline
\end{tabular}


con lo que reportan una TMCAR positiva, contraria a su reducción en la población captada a nivel nacional.

La región Nororiental presenta en 2014 proporciones más altas de uso de tabaco, mariguana y, en un nivel menor, de éxtasis, en comparación con el resto del país; registra TMCAR negativas del consumo de alcohol, cocaína y crack, así como una disminución contraria a la tendencia nacional en el uso de alucinógenos. Por el contrario, muestra uno de los mayores aumentos del uso de metanfetaminas y de éxtasis, así como un incremento del uso de heroína, opuesto a la reducción prevaleciente en la población asistente a tratamiento a nivel nacional. Por otra parte, la población recibida en la región Occidental destaca por un aumento anual relativamente alto de uso de inhalables, así como por la segunda proporción más alta de consumo de metanfetaminas registrada a nivel nacional.

La región Centro presenta porcentajes relativamente altos de uso de inhalables, crack y heroína en comparación con la media nacional; a pesar de la reducida proporción de uso de heroína, ésta registra un aumento anual promedio de más de 33\%. Otras drogas que muestran un fuerte incremento en la región son las metanfetaminas; mientras que las benzodiacepinas y el tabaco observan una de las más fuertes caídas registradas.
En cuanto a la población atendida en la ciudad de México (Distrito Federal), destaca el reporte de un alto consumo de inhalables, crack y, en proporción menor, alucinógenos. De igual modo, se aprecian altas TMCAR de uso de mariguana y alucinógenos, y una disminución del uso de benzodiacepinas.

En la región Centro Sur se aprecia un nivel alto de uso de alcohol y heroína, en comparación con el resto del país, y un crecimiento similar del consumo de mariguana y éxtasis. La región presenta tendencias contrarias a las observadas en el resto de México para el consumo de alucinógenos, cuya utilización disminuye, y de inhalables y heroína, que presentan, en este caso, una TMCAR positiva. Por último, la población captada en tratamiento en unidades del Sur del país reporta niveles relativamente altos de uso de mariguana, cocaína (pese a su tendencia a la baja) y éxtasis; así como una disminución comparativamente alta del consumo de tabaco y un aumento del de inhalables, contrario a la disminución de su uso entre la población admitida en tratamiento a nivel nacional.

\section{DISCUSIÓN Y CONCLUSIONES}

El presente estudio tuvo como objetivo comparar las características sociodemográficas y de consumo de sustancias en usuarios de drogas que solicitan tratamiento,

Tabla 3

Inicio del consumo de sustancias en usuarios de dogas ilícitas solicitantes de tratamiento en Centros de Integración Juvenil en 2007 y 2014

\begin{tabular}{|c|c|c|c|c|}
\hline & & $\begin{array}{c}2007 \\
(N=18,136)\end{array}$ & $\begin{array}{c}2014 \\
(N=21,320)\end{array}$ & Sig. \\
\hline \multicolumn{5}{|l|}{ Edad de inicio del consumo de: } \\
\hline Tabaco & Mediana (rango intercuartil) & 14 años (1.5) & 14 años (1) & $>.001$ \\
\hline Alcohol & Mediana (rango intercuartil) & 15 años (2) & 14 años (1.5) & $>.001$ \\
\hline Drogas ilícitas & Mediana (rango intercuartil) & 16 años (2) & 15 años (2) & $>.001$ \\
\hline \multirow{2}{*}{$\begin{array}{l}\text { Inicio temprano del consumo de } \\
\text { drogas ilícitas }\end{array}$} & Antes de los 13 años & $9.6 \%$ & $12.8 \%$ & $>.001$ \\
\hline & 13 años o más & $90.4 \%$ & $87.2 \%$ & \\
\hline \multirow[t]{10}{*}{ Drogas ilícitas de inicio } & Cannabis & $52.9 \%$ & $73.6 \%$ & $>.001$ \\
\hline & Inhalables & $18.2 \%$ & $12.4 \%$ & \\
\hline & Cocaína & $16.2 \%$ & $6.6 \%$ & \\
\hline & Crack & $6.0 \%$ & $2.0 \%$ & \\
\hline & Metanfetaminas & $1.9 \%$ & $1.9 \%$ & \\
\hline & Éxtasis & $.2 \%$ & $.3 \%$ & \\
\hline & Benzodiacepinas & $1.9 \%$ & $1.7 \%$ & \\
\hline & Alucinógenos & $.3 \%$ & $.2 \%$ & \\
\hline & Heroína & $.2 \%$ & $.2 \%$ & \\
\hline & Otras sustancias & $2.2 \%$ & $1.0 \%$ & \\
\hline
\end{tabular}


Tabla 4

Consumo de sustancias en usuarios de dogas ilícitas solicitantes de tratamiento en Centros de Integración Juvenil en 2007 y 2014

\begin{tabular}{|c|c|c|c|c|c|c|c|c|c|}
\hline & \multicolumn{3}{|c|}{ Consumo alguna vez en la vida } & \multicolumn{3}{|c|}{$\begin{array}{c}\text { Consumo en los } 30 \text { días previos } \\
\text { a la solicitud de tratamiento }\end{array}$} & \multicolumn{3}{|c|}{$\begin{array}{l}\text { Reporte como droga de mayor } \\
\text { impacto }\end{array}$} \\
\hline & $\begin{array}{c}2007 \\
(N=18,136) \\
\end{array}$ & $\begin{array}{c}2014 \\
(N=21,320) \\
\end{array}$ & Sig. * & $\begin{array}{c}2007 \\
(N=18,136)\end{array}$ & $\begin{array}{c}2014 \\
(N=21,320)\end{array}$ & Sig.* & $\begin{array}{c}2007 \\
(N=18,136)\end{array}$ & $\begin{array}{c}2014 \\
(N=21,320) \\
\end{array}$ & Sig. \\
\hline Tabaco & $86.6 \%$ & $83.4 \%$ & $>.001$ & $68.2 \%$ & $59.2 \%$ & $>.001$ & $8.1 \%$ & $6.8 \%$ & $>.001$ \\
\hline Alcohol & $90.4 \%$ & $86.2 \%$ & $>.001$ & $59.0 \%$ & $53.0 \%$ & $>.001$ & $16.1 \%$ & $13.4 \%$ & \\
\hline Cannabis & $70.8 \%$ & $87.1 \%$ & $>.001$ & $28.7 \%$ & $52.7 \%$ & $>.001$ & $15.8 \%$ & $47.4 \%$ & \\
\hline Inhalables & $37.5 \%$ & $31.7 \%$ & $>.001$ & $15.1 \%$ & $13.2 \%$ & $>.001$ & $14.3 \%$ & $11.1 \%$ & \\
\hline Cocaína & $53.9 \%$ & $31.8 \%$ & $>.001$ & $17.0 \%$ & $7.4 \%$ & $>.001$ & $13.5 \%$ & $3.8 \%$ & \\
\hline Crack & $37.7 \%$ & $17.3 \%$ & $>.001$ & $20.1 \%$ & $5.7 \%$ & $>.001$ & $20.2 \%$ & $5.0 \%$ & \\
\hline Metanfetaminas & $13.4 \%$ & $16.0 \%$ & $>.001$ & $5.5 \%$ & $7.2 \%$ & $>.001$ & $7.1 \%$ & $8.0 \%$ & \\
\hline Éxtasis & $4.4 \%$ & $5.9 \%$ & $>.001$ & $.6 \%$ & $1.0 \%$ & $>.001$ & $.3 \%$ & $.3 \%$ & \\
\hline Benzodiacepinas & $16.0 \%$ & $13.1 \%$ & $>.001$ & $3.8 \%$ & $4.3 \%$ & .014 & $1.6 \%$ & $1.7 \%$ & \\
\hline Alucinógenos & $8.6 \%$ & $9.5 \%$ & .002 & $.7 \%$ & $1.1 \%$ & $>.001$ & $.5 \%$ & $.5 \%$ & \\
\hline Heroína & $3.3 \%$ & $2.2 \%$ & $>.001$ & $.9 \%$ & $.7 \%$ & .122 & $1.0 \%$ & $.9 \%$ & \\
\hline
\end{tabular}

* La prueba de diferencias se aplicó para cada sustancia dado que el porcentaje de uso de cada una es independiente del resto.

Tabla 5

Otras variables de consumo en usuarios de drogas ilícitas solicitantes de tratamiento en Centros de Integración Juvenil en 2007 y 2014

\begin{tabular}{|c|c|c|c|c|}
\hline & & $\begin{array}{c}2007 \\
(\mathrm{~N}=18,136)\end{array}$ & $\begin{array}{c}2014 \\
(\mathrm{~N}=21,320)\end{array}$ & Sig. \\
\hline $\begin{array}{l}\text { Uso combinado de su } \\
\text { gas ilícitas) }\end{array}$ & en el último año (incluye alcohol y dro- & $63.9 \%$ & $58.7 \%$ & .000 \\
\hline \multicolumn{5}{|c|}{ Frecuencia del consumo de drogas ilícitas en el último mes } \\
\hline & Menos de una vez a la semana & $23.9 \%$ & $26.1 \%$ & \multirow[t]{4}{*}{.000} \\
\hline & Una o dos veces a la semana & $25.8 \%$ & $21.7 \%$ & \\
\hline & Cada tercer día & $16.6 \%$ & $15.9 \%$ & \\
\hline & Diario & $33.8 \%$ & $36.2 \%$ & \\
\hline \multicolumn{5}{|c|}{$\begin{array}{l}\text { Lapso transcurrido entre el inicio del consumo y la solicitud de trata- } \\
\text { miento en ClJ: }\end{array}$} \\
\hline Tabaco & Mediana (rango intercuartil) & 8 años (6.5) & 4 años (4.5) & .000 \\
\hline Alcohol & Mediana (rango intercuartil) & 8 años (6) & 4 años (4.5) & .000 \\
\hline Drogas ilícitas & Mediana (rango intercuartil) & 5 años (5.5) & 2 años (3.5) & .000 \\
\hline
\end{tabular}

además de contrastar las tendencias de crecimiento en el periodo 2007-2014. Dentro de los principales hallazgos se encontró un incremento generalizado de la población atendida, un aumento en el porcentaje de personas que reportaron consumo de cannabis, y una disminución del reporte de consumo de tabaco y alcohol.

El aumento del uso de mariguana coincide con lo reportado en las encuestas poblacionales en México (Villatoro et al., 2012b; Villatoro et al., 2014). Por el contrario, la disminución del uso de cocaína, crack e inhalables difiere de lo registrado en la población general (Villatoro et al., 2012, 2012b) y entre estudiantes de la Ciudad de México (Villatoro et al., 2014), si bien en este último caso se ha observado una disminución relativa del uso de cocaína entre hombres. Conviene señalar también el aumento del consumo de metanfetaminas, concordante con la Encuesta Nacional de Adicciones, así como la disminución del consumo de heroína entre la población 
Tabla 6

Consumo de sustancias en el mes previo a la solicitud de tratamiento en usuarios de drogas ilícitas solicitantes de tratamiento en Centros de Integración Juvenil en 2007 y 2014, por regiones: porcentajes y tasa media de crecimiento anual real (TMCAR)

\begin{tabular}{|c|c|c|c|c|c|c|c|c|c|c|c|c|}
\hline 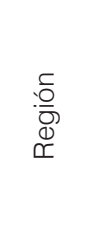 & & 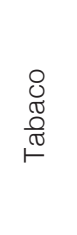 & $\begin{array}{l}\overline{0} \\
\frac{}{0} \\
\frac{0}{<}\end{array}$ & 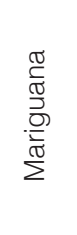 & 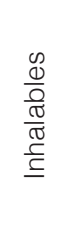 & 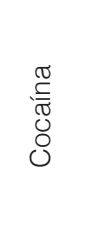 & $\begin{array}{l}\text { U } \\
\frac{\pi}{U}\end{array}$ & 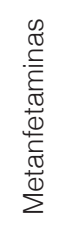 & 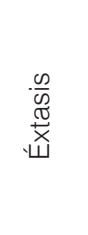 & 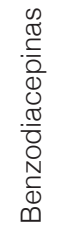 & 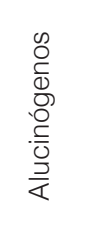 & $\begin{array}{l}\frac{\sigma}{\frac{\sigma}{0}} \\
\frac{0}{0} \\
\frac{0}{1}\end{array}$ \\
\hline \multirow{3}{*}{$\begin{array}{l}\frac{1}{0} \\
\frac{0}{0} \\
\frac{0}{0} \\
\frac{0}{ \pm} \\
\frac{0}{2}\end{array}$} & \% en 2007 & 68.8 & 58.3 & 33.9 & 5.5 & 20.3 & .8 & 30.4 & 1.4 & 6.6 & .5 & 2.8 \\
\hline & \% en 2014 & 58.2 & 49.5 & 50.1 & 2.7 & 9.1 & .3 & 23.1 & .6 & 5.8 & .2 & 1.4 \\
\hline & TMCAR (\%) & -2.3 & -2.3 & 5.7 & -9.6 & -10.8 & -13.2 & -3.8 & -10.9 & -1.8 & -10.9 & -9.0 \\
\hline \multirow{3}{*}{$\begin{array}{l}\frac{1}{\bar{D}} \\
\frac{0}{0} \\
\frac{0}{2}\end{array}$} & \% en 2007 & 69.5 & 63.7 & 30.7 & 12.8 & 32.5 & 9.4 & 6.3 & .3 & 4.1 & .1 & 4.4 \\
\hline & \% en 2014 & 63.1 & 55.7 & 56.2 & 17.7 & 7.7 & 1.8 & 6.4 & 1.3 & 9.7 & 1.0 & 2.0 \\
\hline & TMCAR (\%) & -1.4 & -1.9 & 9.0 & 4.7 & -18.6 & -20.8 & .2 & 21.1 & 13.1 & 33.7 & -10.4 \\
\hline \multirow{3}{*}{$\begin{array}{l}\frac{1}{\frac{1}{0}} \\
\frac{0}{0} \\
\frac{0}{2} \\
\frac{\pi}{2}\end{array}$} & \% en 2007 & 74.0 & 67.9 & 31.9 & 18.0 & 20.7 & 35.4 & .2 & .4 & 3.4 & .7 & .1 \\
\hline & \% en 2014 & 64.5 & 55.6 & 61.0 & 15.3 & 5.9 & 2.7 & 1.4 & 1.6 & 6.9 & .6 & .1 \\
\hline & TMCAR (\%) & -1.9 & -2.8 & 9.7 & -2.3 & -16.5 & -30.8 & 31.6 & 21.1 & 10.8 & -2.4 & 2.7 \\
\hline \multirow{3}{*}{$\begin{array}{l}\frac{1}{0} \\
\frac{0}{U} \\
\frac{0}{0} \\
0\end{array}$} & \% en 2007 & 70.9 & 59.4 & 27.8 & 7.5 & 20.6 & 17.2 & 6.9 & .5 & 4.1 & .6 & .4 \\
\hline & \% en 2014 & 62.5 & 53.9 & 49.4 & 12.3 & 7.6 & 3.9 & 14.3 & .9 & 5.7 & .9 & .4 \\
\hline & TMCAR (\%) & -1.8 & -1.4 & 8.6 & 7.3 & -13.2 & -19.1 & 10.9 & 9.6 & 4.8 & 6.2 & -2.0 \\
\hline \multirow{3}{*}{ 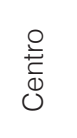 } & \% en 2007 & 70.3 & 58.7 & 27.8 & 19.8 & 12.3 & 21.1 & 1.1 & .5 & 3.5 & 1.0 & .1 \\
\hline & \% en 2014 & 58.2 & 52.2 & 50.2 & 16.7 & 5.8 & 7.1 & 3.9 & .8 & 1.8 & 1.3 & 1.1 \\
\hline & TMCAR (\%) & -2.7 & -1.7 & 8.8 & -2.4 & -10.1 & -14.4 & 19.3 & 6.3 & -8.7 & 3.6 & 33.1 \\
\hline \multirow{3}{*}{ 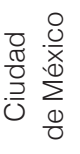 } & \% en 2007 & 64.1 & 53.5 & 24.9 & 23.1 & 8.8 & 26.1 & .5 & .8 & 2.7 & .7 & .1 \\
\hline & \% en 2014 & 57.6 & 52.6 & 52.1 & 16.1 & 7.5 & 10.7 & .9 & 1.3 & 1.7 & 1.7 & - \\
\hline & TMCAR (\%) & -1.5 & -.2 & 11.1 & -5.0 & -2.3 & -12.0 & 9.6 & 7.8 & -6.7 & 13.6 & - \\
\hline \multirow{3}{*}{ 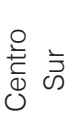 } & \% en 2007 & 57.7 & 58.7 & 27.0 & 8.6 & 25.7 & 15.8 & 2.0 & .2 & 4.6 & .9 & 1.0 \\
\hline & \% en 2014 & 56.8 & 56.0 & 55.5 & 11.6 & 7.8 & 3.4 & 4.9 & .7 & 7.8 & .9 & 1.2 \\
\hline & TMCAR (\%) & -.2 & -.7 & 10.8 & 4.4 & -15.6 & -19.5 & 13.8 & 21.0 & 7.7 & -1.3 & 2.4 \\
\hline \multirow{3}{*}{ う } & \% en 2007 & 69.3 & 64.6 & 32.7 & 7.6 & 18.3 & 34.8 & .3 & .7 & 2.4 & .5 & .1 \\
\hline & \% en 2014 & 57.0 & 54.3 & 61.8 & 9.6 & 9.5 & 8.2 & .8 & 1.4 & 3.6 & 1.3 & - \\
\hline & TMCAR (\%) & -2.8 & -2.4 & 9.5 & 3.4 & -9.0 & -18.7 & 12.4 & 11.0 & 6.1 & 14.1 & - \\
\hline
\end{tabular}

recibida en tratamiento tanto a nivel nacional como en las regiones donde de manera histórica se han registrado niveles relativamente más altos.

Aunque el perfil sociodemográfico de la población atendida resulta similar en varios puntos a lo reportado en otros sitios (Marín-Navarrete et al., 2014), deben tenerse presentes los cambios observados, entre ellos el aumento en el consumo por parte de mujeres y menores de edad, y el incremento de la población que reporta un inicio temprano del uso de sustancias; no se debe obviar el aumento de la frecuencia del uso de drogas ilícitas en un lapso reciente (último mes) ni la reducción de la edad de inicio y el lapso transcurrido entre el primer consumo y la búsqueda de tratamiento.

Estos cambios adquieren relevancia para efectos de diseño y aplicación de programas y servicios, tanto en materia de prevención como de tratamiento del uso de sustancias; resalta la relevancia de contar con alternativas de atención con perspectiva de género, ajustadas a las necesidades de jóvenes adolescentes y dirigidas a frenar, en una etapa temprana, el proceso adictivo.

En cuanto a la comparación del uso de sustancias y las tendencias observables en la población atendida en las distintas regiones del país, el presente estudio indi- 
ca la importancia de establecer programas y servicios que consideren las diferencias detectadas. Entre éstas cabe subrayar el aumento del uso de heroína en la población atendida en las regiones Centro y Centro Sur, dato que resulta de especial relevancia para la planeación de servicios especializados y de programas de sustitución para usuarios de esta sustancia. En el mismo sentido, es relevante destacar el alto consumo de metanfetaminas en las regiones Noroccidental y Occidental -sin obviar que su uso está en aumento entre la población solicitante de tratamiento en la mayor parte de las regiones del país-. Asimismo, debe destacarse el nivel relativamente alto de consumo de inhalables que aún persiste entre la población captada en la Ciudad de México y en las regiones Norcentral y Centro; así como el aumento del uso de alucinógenos y éxtasis, sobre todo en población atendida en la Ciudad de México y en las regiones Norcentral, Centro Sur y Sur.

En cuanto a las implicaciones para la investigación de los resultados presentados, resulta viable plantearse diversas interrogantes respecto a los cambios registrados en el patrón de consumo, en términos, por ejemplo, de su posible relación con modificaciones en la accesibilidad de las sustancias, de sus efectos o su potencial adictivo, entre otras. También cabe preguntarse en qué medida estas transformaciones están vinculadas con las observadas en el perfil social y demográfico de la población solicitante de atención terapéutica, tales como el aumento de mujeres y de la población de menor de edad. De igual modo, es necesario estudiar los factores del entorno social y de ajuste psicosocial que afectan a la población potencialmente usuaria de servicios y que podrían estar influyendo en las características que está adquiriendo el problema.

Entre las limitaciones del presente estudio se encuentra la dificultad para generalizar los hallazgos, si se consideran las características, la cobertura, la ubicación y el costo de la atención, lo que implica la posibilidad de que las características particulares de los usuarios puedan no replicarse en otros centros públicos o privados. Otra de las limitaciones se relaciona con la naturaleza descriptiva de la información: debido a que los datos provienen de un sistema de monitoreo epidemiológico, los mecanismos causales, tanto del crecimiento de la demanda como de los cambios en el perfil y el patrón de consumo de la población atendida, son desconocidos. En cuanto a lo primero, futuros estudios deberán centrarse en el establecimiento de relaciones causales específicas entre variables como cobertura, situación geográfica, promoción y percepción de la utilidad de los servicios, con la finalidad de determinar los mecanismos relacionados con el incremento de la demanda de atención.
Los hallazgos del presente estudio reflejan, sin embargo, la importancia de la información epidemiológica obtenida con población que busca servicios de atención especializada, no sólo como una fuente útil para apoyar el diseño, la planeación y el ajuste de programas y servicios, sino también como un medio para ampliar el conocimiento del comportamiento del consumo de drogas en el país.

\section{FUENTES DE FINANCIAMIENTO}

No se recibió financiamiento específico para el levantamiento de datos, análisis o preparación del presente manuscrito.

\section{CONFLICTOS DE INTERÉS}

Los autores laboran en Centros de Integración Juvenil A.C., sin embargo, el contenido del artículo representa su opinión y no la de esta institución.

\section{AGRADECIMIENTOS}

Los autores agradecen el apoyo de Alejandro X. Álvarez Gutiérrez en la preparación de este trabajo.

\section{REFERENCIAS}

Centros de Integración Juvenil. (2015). Sistema de Información Epidemiológica del Consumo de Drogas. México: Centros de Integración Juvenil, Dirección de Investigación y Enseñanza, Subdirección de Investigación.

Clarac, P., \& Wagner, F. (1990). Análisis de algunas variables en una muestra de pacientes farmacodependientes 1986. En F. Wagner y R. Ortiz (comp.), Estudios acerca de la farmacodependencia en México realizados por Paule Clarac (pp. 111-126). México: Centros de Integración Juvenil.

Díaz, D., Sánchez, R., \& Guisa, V. (2004). Registro y estudio de casos en centros de tratamiento especializado. En M.E. Medina-Mora (coord.), Observatorio mexicano en tabaco, alcohol y otras drogas, Metodología para la elaboración de estudios epidemiológicos a nivel nacional y local y estudios para grupos especiales relacionados con las adicciones (pp. 73-77). México: Consejo Nacional Contra las Adicciones.

Faller, S., Peuker, A., Sordi, A., Stolf, A., Souza-Formigoni, M., Santos, M.,... Kessler, F. (2014). Who seeks public treatment for substance abuse in Brazil? Results of a multicenter study involving four Brazilian state capitals. Trends of Psychiatry and Psychotherapy, 36(4), 193-202.

Marín-Navarrete, R., Templos Núñez, L., Eliosa Hernández, A., Villalobos Gallegos, L., Fernández Mondragón, J., Pérez López, A.,... Horigian, V. (2014). Characteristics of a treatment-seeking population in outpatient addiction treatment centers in 
Mexico. Substance Use \& Misuse, Early Online, 1-11. DOI: 10.3109/10826084.2014.931972

Ponizovsky, A., Rosca, P., Haklai, Z., \& Goldberger, N. (2015). Trends in dual diagnosis of severe mental illnes and substance use disorders, 1996-2010, Israel. Drug and Alcohol Dependence, 148, 203-208.

Programa de las Naciones Unidas para la Fiscalización Internacional de Drogas. (2003). Elaboración de un sistema integrado de información sobre las drogas. Módulo 1. Viena: Oficina de las Naciones Unidas contra la Droga y el Delito.

Programa Mundial de Evaluación del Uso Indebido de Drogas, Observatorio Europeo de las Drogas y las Toxicomanías. (2006). Orientaciones para la medición de la demanda de tratamiento de drogas. Módulo 8. Viena: Oficina de las Naciones Unidas contra la Droga y el Delito.

Sánchez, R. \& López, L. (1985). Características demográficas y patrones de inicio en el consumo de drogas en asistentes a los cen- tros locales, recuperación de los archivos de siete años (19761982). México: Centros de Integración Juvenil (reporte interno).

Villatoro, J., Medina-Mora, M.E., Fleiz, C., Téllez, M., Mendoza, L., Romero, M.,...Guisa, V. (2012 a). Encuesta Nacional de Adicciones 2011: Reporte de drogas. México: Instituto Nacional de Psiquiatría Ramón de la Fuente Muñiz; Instituto Nacional de Salud Pública; Secretaría de Salud.

Villatoro, J., Medina-Mora, M.E., Fleiz, C., Moreno, M., Robles, N., Bustos, M.,... Amador, N. (2012 b). El consumo de drogas en México: Resultados de la Encuesta Nacional de Adicciones, 2011. Salud Mental, 35(6), 447-457.

Villatoro, J., Mendoza, M.., Moreno, M., Robles, N., Fregoso, D., Bustos, M., ... Medina-Mora, M.E. (2014). Tendencias del uso de drogas en la Ciudad de México: Encuesta de estudiantes, octubre 2012. Salud Mental, 37(5), 423-435. 